\title{
SOCIALIZAÇAO E DEPRESSÃO INFANTIL
}

\section{SOCIALIZATION AND CHILDHOOD DEPRESSION}

\author{
Roseli Schultz Chiovitti Cavalcante ${ }^{1}$
}

CAVALCANTE, R. S. C. Socialização e Depressão infantil. Rev. Bras. Cresc. Desenv. Hum., São Paulo, 6(1/2), 1996.

\begin{abstract}
Resumo: A depressão infantil e a depressão em adultos tendem a se manifestar de maneiras semelhantes. Além dos problemas emocionais e fisicos que a depressão causa, esta pode também interferir na competência social das crianças deprimidas. Por sua vez, estes problemas sociais podem exacerbar os sintomas depressivos além de causar problemas adicionais.Assim sendo, é importante que profissionais da saúde e educação estejam preparados para intervir junto às crianças deprimidas com as quais entrem em contato. Este artigo não somente discute as repercussões que a depressão pode ter na vida e desenvolvimento social normal da criança, como também sugere intervenções práticas que educadores e terapeutas podem implementar para amenizar os efeitos desta psicopatologia e prevenir uma socialização inadequada.
\end{abstract}

Palavras-clave: depressão infantil, competência social, modelos terapêuticos, cognição social, socialização.

Só recentemente, profissionais e pesquisadores das áreas de saúde e educação têm começado a entender os problemas da socialização e das interações sociais encontrados por crianças que sofrem de depressão, e como estes contribuem para o aparecimento e extensão de sua psicopatologia. Na verdade, só pouco tempo atrás um consenso foi alcançado sobre a exitência da depressão infantil como uma psicopatologia distinta. LAPOUSE (1966) acreditava que a sintomatologia depressiva era característica do desenvolvimento normal da criança, especialmente durante a adolescência. Esta hipótese foi rejeitada após ter-se constatado que não são todas as crianças que durante o seu desenvolvimento normal apresentam simtomatologia depressiva. Outra linha teórica, conhecida como "equivalentes depressivos", foi desenvolvida nos anos sessenta e afirmava que a manifestação da depressão na criança era diferente da sua manifestação em adultos (GLASER, 1968; TOOLAN, 1962). De acordo com este modelo teórico, não haveria uma simtomatologia clara e característica da depres- são em crianças. Comportamento agressivo e antisocial, enurese e somatização poderiam ser todos interpretados como sinais de uma depressão subjacente, sendo esta chamada "depressão mascarada”. Esta teoria provocou controvérsias entre clínicos e pesquisadores. No entanto, estudos subseqüentes demonstraram que muitas crianças que sofrem daqueles problemas não possuem uma depressão latente. Atualmente, acredita-se que a depressão infantil se inanifesta de uma maneira similai à depressão dos adultos (CARLSON \& CANTWELL, 1980; KAZDIN, 1990; REYNOLDS, 1985). No entanto, embora as similaridades entre a depressão adulta e infantil sejam importantes, as diferenças devidas ao desenvolvimento devem ser cuidadosamente consideradas no processo diagnóstico (KAZDIN, 1990).

Por apresentar um critério confiável para o diagnóstico dessa psicopatologia, a depressão, o instrumento mais usado é o DSM-IV (Diagnostic and Statistical Manual of Mental Disorders IV). O DMS-IV define a depresssão como uma disposição deprimida ou irritável e perda de interesse

1 Doutoranda em Psicologia Educacional e Escolar pela Indiana University. End.: Indiana University. Banta \# F 124. Bloomington, IN 47408. United States of America. Tel: (812) 857-2414 Fax: (812) 855-7462. 
em todas ou quase todas as atividades, por um período de ao menos duas semanas, acompanhado de pelo menos quatro sintomas associados, como por exemplo, mudanças no apetite ou peso, dificuldade de concentração, agitação ou retardamento psicomotor, menos energia e disposição, autodepreciacão, culpa excessiva e pensamentos freqüentes sobre morte e suicídio. O diagnóstico da depressão deve ser feito somente se causas orgânicas que possam iniciar ou manter o problema não forem aparentes, ou se esses sintomas não forem uma reação normal ao luto (DSM-IV, 1994).

\section{IMPORTÂNCIA DAS EXPERIÊNCIAS SOCIAIS NO DESENVOLVIMENTO}

Estudos feitos na área do desenvolvimento infantil têm sugerido que as interações sociais recíprocas contribuem de modo único para o desenvolvimento emocional, assim como para a definição da identidade da criança como ser social (HARTUP, 1989). É no contexto das interações sociais que a criança desenvolve a linguagem, coordena suas ações com as dos outros, e desenvolve o conhecimento de si mesma e do mundo. HARTUP (1983) sugere que existem dois tipos de interações sociais, as quais são necessárias para o desenvolvimento saudável da criança: o primeiro tipo consiste dos relacionamentos verticais que incluem o relacionamento da criança com os pais ou responsáveis; o segundo, que envolve os relacionamentos horizontais, consiste, por sua vez, da interação da criança com os seus colegas e amigos.

As experiências da criança com os colegas são importantes porque a ajudam a desenvolver as habilidades sociais necessárias para engajar-se em interações e relacionamentos positivos, assim como para desenvolver o seu senso de valor próprio. Piaget (YOUNISS, 1980) foi um forte propagador desta idéia. Ele afirmava que os conflitos que ocorrem no relacionamento infantil são importantes para o desenvolvimento da moralidade e das interações colaborativas. Alguns trabalhos mais recentes têm sugerido também que as interações sociais com os colegas criam na criança um senso de segurança. DOUVAN e ADELSON (1966) por exemplo, propuseram que durante a pré-adolescência e adolescência, a criança precisa ter o suporte dos amigos para que as ansiedades e medos causados pelas mudanças corporais e emocionais, que ocorrem durante essa fase, sejam reduzidos. FINE (1981) propôs que as interações sociais com os amigos e colegas, promovem um ambiente seguro no qual novos modelos de expressão social podem ser testados, criando oportunidades para que a criança adquira e pratique novos comportamentos sociais.

Alguns trabalhos têm demonstrado que as experiências sociais com outras crianças também contribuem para o desenvolvimento do auto-conceito. Sullivan (YOUNISS, 1980), em particular, enfatizou a importância das relações socias durante a idade escolar e adolescência para o desenvolvimento de um autoconceito saudável. As evidências apresentadas acima mostram que as interações sociais recíprocas horizontais ajudam a criança a se desenvolver tanto emocionalmente como socialmente. Por outro lado, nos meios científicos aceita-se atualmente que problemas no estabelecimento de interações sociais recíprocas saudáveis podem contribuir para o aparecimento e manutenção de psicopatologias, incluíndo a depressão infantil (CLARIZIO, 1985; EWINSON, 1974).

\section{EFEITOS DA DEPRESSÃO NA SOCIALIZAÇÃO DA CR1ANÇA}

Uma das teorias cognitivas mais importantes que explica a etiologia dos processos depressivos é a teoria cognitiva de BECK (1970). Ele argumenta que estruturas cognitivas ou esquemas afetarn a codificação, o armazenamento e o acesso à informação no cérebro. Ele teoriza que indivíduos depressivos possuem esquemas mentais com tendências negativas, o que os leva a interpretar de uma maneira equivocada os estímulos sociais que recebem, eliminando as informações positivas e exagerando nas informações negativas. Estes erros cognitivos levam a criança deprimida a ter uma visão negativa de si mesma, do seu ambiente e do futuro.

Um trabalho desenvolvido por DODGE et al. (1992) confirmou que, de fato, crianças deprimidas apresentam deficiências cognitivas que interferem no seu funcionamento na área das interações sociais. Estes autores concluíram que elas têm, geralmente, percepções equivocadas. Além disso, crianças deprimidas tendem a ser socialmente passivas porque acreditam que serão punidas ou rejeitadas se forem mais ativas. Acreditase que este estilo de interação disfuncional leve ao isolamento social do indivíduo (rejeição). Conseqüentemente, cognições negativas sobre si mesmo e falta de autoconfiança podem se exacerbar.

Sabe-se que adultos deprimidos são comumente menos ativos, quase não sorriem, e relatam desconforto em situações sociais (GOTLIB ROBINSON, 1982; JACOBSON \& ANDERSON, 1982). Embora pouco tenha sido escrito sobre o comportamento social das crianças depri- 
midas, acredita-se que elas apresentem problemas sociais semelhantes. Um estudo conduzido por ALTMAN \& GOTLIB (1988) dá suporte a esta idéia. Eles observaram crianças deprimidas durante o recreio escolar e notaram que elas passavam mais tempo sozinhas, além de se engajarem mais freqüentemente em interações negativas do que seus colegas não deprimidos.

Se não for tratada, esta falta de habilidade na área social poderá levar a criança a desenvolver problemas adicionais, como: mau desempenho escolar, repertência, problemas de ajustamento, delinquência juvenil, comportamento criminoso e piora do quadro psicopatológico (ASHER, 1987; HERSH \& WALKER, 1983; PARKER \& ASHER, 1987). Além do mais, se as perspectivas de Piaget e Sullivan estiverem corretas, crianças deprimidas poderão também sofrer danos permanentes devido às suas interações sociais inapropriadas, como por exemplo, dificuldade em estabelecer um senso moral adequado e participar efetivamente em atividades de grupo. A discussão apresentada acima deixa claro que crianças deprimidas se beneficiariam de um programa terapêutico que as estimulassem a se relacionarem mais positivamente, assim como a agirem de maneira mais apropriada ao seu contexto social.

\section{APRENDENDO A SE RELACIONAR}

Os programas terapêuticos de intervenção nessa área são geralmente planejados com o intuito de alterar os comportamentos interpessoais inapropriados ao ambiente do indivíduo, assim como alterar suas cognições distorcidas associadas a esses comportamentos. De acordo com GOLDSTEIN et al. (1980), uma socialização adequada ocorre quando a crianca tem: “(1) a habilidade de organizar cognições e comportamentos em um curso de ação integrado e direcionado a objetivos culturalmente apropriados; e (2) a habilidade de continuamente avaliar e modificar seu comportamento para que alcance esses objetivos.”

Além do mais, estudos têm demonstrado que a incompetência social pode ser devida a problemas na aquisição ou na execução dos comportamentos sociais apropriados (GRESHAM, 1994). Problemas de aquisição ocorrem quando a criança não aprendeu os comportamentos necessários para se engajar em interações sociais apropriadas. No entanto, no caso de problemas de desempenho, o indivíduo conhece os comportamentos sociais adequados, mas é incapaz de executá-los quando a situação se apresenta. Crianças deprimidas podem apresentar tantos problemas de aqui- sição como de desempenho. Assim sendo, é importante para o clínico ou educador, distinguir o tipo de deficiência que a criança apresenta para que possa recomendar e implementar, junto a ela, um tratamento efetivo (Ib., 1994).

A maioria das intervenções disponíveis atualmente tem em comum as premissas de que comportamentos sociais: (1) são aprendidos através de observações do comportamento dos outros, demonstrações, prática, e "feed-back"; (2) envolvem comportamentos verbais e não-verbais; (3) incluem não somente a iniciação efetiva das interações, assim como respostas apropriadas; (4) são interativos; e (5) são interativos; e (5) são influenciados pelo ambiente no qual a pessoa se encontra (MICHELSON et al., 1983; apud ELLIOT \& ERSHLER, 1994). A seguir exporemos cada um dos diferentes modelos terapêuticos.

\section{INTERVENÇÕES BEHAVIORISTAS}

Os procedimentos derivados da teoria do condicionamento operante (behaviorista) tendem a focalizar a intervenção nos comportamentos observáveis, assim como nos antecedentes e conseqüentes destes comportamentos. Assim sendo, comportamentos inapropriados são controlados e modificados através da manipulação das suas conseqüências e também dos fatores que os antecedem (ELLIOT \& ERSHLER, 1994). Presume-se que a criança experimenta problemas nas suas interações interpessoais porque o seu ambiente não é estruturado de uma maneira que promova e facilite comportamentos sociais mais positivos. Uma das técnicas baseada neste modelo psicólogico consiste no treinamento dos colegas para que iniciem junto à criança interações sociais mais positivas. Crianças deprimidas são geralmente isoladas no seu ambiente porque têm dificuldade em iniciar interações positivas. Este método cria assim, oportunidades para que elas participem de interações sociais mais gratificantes as quais, em si mesmas, serão um estímulo para que procure novos contatos sociais. Outra técnica baseada na teoria do condicionamento operante consiste em reforçar a criança quando esta exibir comportamentos sociais apropriados, até que estes se tornem parte do seu repertório efetivo. Ainda outro método é o programa SORC desenvolvido por Kanter e Phillips (apud GRESHAM, 1994) o qual consiste não só na apresentação de reforços, mas também na manipulação dos fatores que antecedem o comportamento e seus processos mediadores (pensamentos, emoções, etc.). Os métodos baseados na teoria do condicionamento operante são mais apropriados no tratamento de crianças 
socialmente isoladas, e que apresentem problemas de desempenho. Isto porque, estes métodos não ensinam a elas novos modelos de comportamento, mas somente reforçam aqueles que elas já possuem em seu repertório.

A segunda categoria de intervenções é baseada na teoria do aprendizado social desenvolvida por Bandura. De acordo com esta perspectiva, comportamentos sociais são adquiridos através da observação do comportamento dos outros, assim como do reforço de comportamentos culturalmente apropriados (ELLIOT \& ERSHLER, 1994). Os métodos desenvolvidos baseados neste modelo teórico enfatizam a demonstração e o “roleplay”. Além do mais, não pressupõe, como no modelo anterior, que a criança já possua as competências sociais, mas sim que precisa aprendê-las. Por isso, estes métodos são úteis, não somente no tratamento das crianças com problemas de desempenho, mas também daquelas com problemas de aquisição.

\section{INTERVENÇÕES \\ COGNITIVO-COMPORTAMENTAIS}

A terceira categoria terapêutica é baseada em teorias cognitivo-comportamentais (ELLIOT \& ERSHLER, 1994). Essas técnicas enfatizam a capacidade da criança para identificar problemas, assim como achar e selecionar soluções apropriadas. Baseado no fato de que a maioria das crianças deprimidas têm sérios problemas na área da cognição social (DODGE et al., 1992), terapias que promovam mudanças nas suas percepções sociais distorcidas, assim como modificações no seu comportamento, parecem ser as mais apropriadas.

A maioria das terapias disponíveis atualmente, envolve a combinação de duas ou mais das técnicas descritas acima. Um modelo eclético que se tem mostrado eficaz no tratamento de indivíduos com problemas na área social é o "Skill Streaming Program” desenvolvido por Goldstein (MACGINNIS \& GOLDSTEIN, 1984). Trata-se de uma intervenção psicoeducacional direcionada a ensinar comportamentos pró-sociais, assim como facilitar o desempenho de tais comportamentos. $\mathrm{O}$ modelo inclui instrução, demonstração, "role-play" e avaliação. Além de ensinar comportamentos so- ciais mais apropriados, o programa procura mudar as percepções inacuradas que as crianças têm do seu ambiente e de si mesmas. Um trabalho desenvolvido por REED (1994), demonstrou que o método de Goldstein é eficaz não só no ensino de novas habilidades sociais, mas também na redução de sintomas depressivos em adolescentes.

\section{LIMITAÇÕES TERAPÊUTICAS}

Deve-se notar aqui que, embora por um lado estes programas terapêuticos produzam resultados positivos, por outro lado possuem também algumas limitações. Os maiores problemas estão relacionados com a generalização e com a manutenção dos comportamentos aprendidos em terapia.

DUPAUL \& ECKERT (1994) identificaram alguns fatores que podem causar este problema. Primeiro, pode ser que tenha ocorrido um erro diagnóstico. Assim sendo, a criança poderá ter sido tratada como supostamente tendo um problema de aquisição, quando de fato o seu problema estaria na área de desempenho (ou vice-versa).

Um segundo fator seria o fato de o clínico responsável pelo tratamento não ter focalizado a intervenção nos comportamentos em que a criança é realmente deficiente. Além do mais, se a cnança tiver sido instruída a usar coinportamentos não apropriados à sua cultura ou ao seu ambiente natural, será mínima a probabilidade de ela empregar os comportamentos ensinados quando a situação se apresentar. Assim sendo, é importante que haja um tratamento individualizado adaptado às condições de vida da criança.

Stokes e Osnes (apud DUPAUL \& ECKERT, 1994) mencionam algumas estratégias que podem ser usadas por profissionais da saúde para promover a generalização e manutenção dos comportamentos socias desejados: (1) identifique e modifique as contigências ambientais que possam contribuir para a manutenção de comportamentos indesejáveis e que impeçam o estabelecimento de comportamentos mais apropriados; (2) inclua na terapia exemplos práticos de comportamentos que a criança possa usar na escola e em outras situações sociais; (3) incorpore na situação terapêutica estímulos sociais e fisicos que normalmente estão presentes no ambiente natural da criança.

\begin{abstract}
The depression in adults and in children usually have similar manifestations. However, in addition to causing physical and emotional distress, childhood depression can also interfere with both children's norrnal social development and their ability to perform in social situations. This, as education and mental health professionals enter in contact with depressed children, it is important for them to be prepared to address these children's specific needs. This article discusses the impact that depression might have in the healthy social development of children. Furthemore,
\end{abstract}


it presents and discusses several social skills training programs that are used to help depressed children to develop better social skills and to avoid the problems of a faulty socialization.

Key-words: childhood depression, social skills, social skills training programs, social cognition, socialization.

\section{REFERÊNCIAS BIBLIOGRÀFICAS}

ALTMAN, E. O.; GOTLIB, I. H. The social behavior of depressed children: An observational study. J. Abnorm. Child Psychol., 16:,2944,1988.

AMERICAN PSYCHIATRIC ASSOCIATION. Diagnostic and statistical manual of mental disorders, $4^{\text {th }}$ ed.Washington, DC, Author, 1994.

ASHER, S. R. Children's peer relations. In: Lamb, M. E. ed., Social and personality development. New York, Holt, Rinehart, and Winston, 1987, p. 91-113.

BECK, A. T. Depression: causes and treatment. Philadelphia, University of Pennsivania Press, 1970.

BECK, A. T.; RUSH, J. A.; SHAW, B. G.; EMERY, G. The cognitive therapy of depression. New York,Guilford Press, 1979.

CARLSON, G. A.; CANTWEEL, D. P. Unmasking masked depression in children and adolescents. Amer. J. Psych., 137: 445-449, 1980.

CLARIZIO, H. F. Cognitive-behavioral treatment of childhood depression. Psychol. Sch., 22: 308-322, 1985.

DODGE, K. A.; QUIGGLE, N. L.; GARBER, J.; PANAK, W. F. Social information processing in aggressive and depressed children. Child Dev., 63: 1305-1320, 1992.

DOUVAN, E.; ANDELSON, J. The adolescence experience. NewYork,Wiley,1966.

DUPAUL, G. J.; ECKERT, T. L. The effects of social skills curricula: Now you see them, now you don't. Sch. Psychol. Quart., 9:113-132, 1994.

ELLIOT, S.N.; ERSHLER, J. Best practices in preschool social skills training. In: THOMAS, A.; GRIMES, J. Best practices in school psychology II, p.591-606. Washington DC, National Association of School Psychologists, 1994.

FINE, G. A. Friends, impression management, and preadolescent behavior. In: ASHER, S. R.; GOTTMAN, J. M. eds. The development of childrens friendships. New York, Cambridge University Press, 1981. p. 29-52.

GLASER, K. Masked depression in children and adolescents. manual Progress in Child Psychiatry and Child Development, I: 345-355, 1968.
GOLDSTEIN, A. P.; SPRAFKIN, R. P.; GERSHAW, N. J.; KLEIN, P. Skill streaming the adolescent. Champaign, IL, ResearchPress, 1980.

GOTLIB, I. H.; ROBINSON, L. A. Response to depressed individuals:discrepancies between self-report and observer-rated behavior. $J$. bnorm. Psychol., 91: 231-240, 1982.

GRESHAM, F. M. Best practices in social skills training. In: THOMAS, A; GRIMES, J. eds., Best practices in school psychology II. Washigton, DC, National Association of School Psychologists,1994. p. 695-710.

HARTUP, W. W. Social relationships and their developmental significance. Amer. Psychol., 44:120-126, 1989.

HARTUP, W. W. Peer realtions. In: HETHERINGTON, E. M.; MUSSEN, P. H. ed. Handbook of childpsychology: v. 4. socialization, personality, and social developement. NewYork, Wiley, 1983 p. 103-196.

HERSH, R.; WALKER, H. Great expectations: making schools effective for all students. Policy Stud. Rev., 2: 147-188, 1983.

JACOBSON, N. S.; ANDERSON, E. A. Interpersonal skills and depression in college students: an analysis of the timimg of self-disclosures. Behav. Therapy, 13: 271-282,1982.

KAZDIN, A.E. Childhood depression. Child Psychol. Psych, 31: 121-160, 1990.

LAPOUSE, R. The epidemiology of behaviour disorders in children. J. Affect. Disord. Child., 3: 594-599, 1966.

LEWINSON, P. M. Clinical and theoretical aspects of depression. In: CALHOUN,K.S.; ADAMS, H.E.; MITCHELL, K.M. eds., $l n-$ novative treatment methods of psychopathology. New York, Wiley,1974. p.63- 120.

MCGINNIS, E.; GOLDSTEIN, A. P., Skillstreaming the elementary school child. Champaign, IL Research Press, 1984.

PARKER, J.; ASHER, S. Peer relations and later personal adjustment: Are low-accepted children at risk? Psychol. Bu11.,102: 357-389, 1987.

REED, M.K. Social skills training to reduce depression in adolescents. Adolesc., 29: 293302,1994. 
REYNOLDS, W.M. Depression in childhood and adolescence: Diagnosis, assessment, intervention strategies and research. In: KRATOCHWILL, T. R. Advances in school psychology. New Jersey, Lawrence Erlbaum, 1985. v.4 p. 133-189.

TOOLAN, J. M. Depression in children and adolescents. Amer. J. Orthpsych., 32: 404-414, 1962.

YOUNISS, J. Parents and peers in social development: A Sullivan-Piaget perspective. Chicago, The University of ChicagoPress, 1980.

Recebido em: 29/04/97

Aprovado em: 20/05/97 